\title{
Planar Doppler velocimetry measurements of flows using imaging fibre bundles
}

\author{
David S. Nobes, Helen D. Ford and Ralph P. Tatam ${ }^{1}$ \\ Optical Sensors Group, Centre for optcal photonics and engineering School of Engineering, \\ Cranfield University, Cranfield, Bedford, UK, MK43 0AL
}

\begin{abstract}
The development of a planar Doppler velocimetry is described. The technique is capable of measuring the three, instantaneous components of velocity in two dimensions using a single pair of signal and reference cameras. PDV can be used to measure the instantaneous 3-D velocity of a fluid by using an absorption line filter (ALF) to determine the Doppler shifted frequency of a narrow line pulsed laser (Nd:YAG) that has been scattered off particles seeded into the flow. The velocity of the fluid is determined using the Doppler formula and is dependent on the laser direction and the viewing direction. Hence, only one velocity component of the flow is measured. This component can be measured in two spatial dimensions using an array detector such as a CCD camera. To capture the three components, three such measurement heads have been used viewing from different angles. In the technique presented here the three views are ported from the collection optics to a single imaging plane using flexible fibre imaging bundles. These are made up of a coherent array of single fibres and are combined at one end as the input plane to the measurement head. The paper discusses the issues involved in developing a full three-dimensional velocity measurement system.
\end{abstract}

Keywords: Planar Doppler velocimetry, imaging fibre bundles, flow velocity measurement.

\section{INTRODUCTION}

The theory of planar Doppler velocimetry (PDV) is based on the Doppler principle that light, when scattered from a moving object is frequency shifted depending on the velocity of that object. The Doppler formula describes the relationship between the velocity of the object and the frequency shift of the light scattered from that object and can be expressed using the vector equation

$$
\Delta \boldsymbol{v}=\frac{\boldsymbol{v}_{0}}{\mathrm{c}}(\hat{\mathbf{o}}-\hat{\mathbf{i}}) \bullet \mathbf{V}
$$

Here, $\Delta v=v_{\mathrm{D}}-v_{\mathrm{o}}$ is the difference between the Doppler shifted frequency $\left(v_{\mathrm{D}}\right)$ and the frequency of the light source $\left(v_{0}\right), \mathbf{c}$ is the free space speed of light, $\mathbf{V}$ is the velocity vector of the object that is scattering the light, $\hat{\mathbf{o}}$ the unit direction vector to the viewer and $\hat{\mathbf{i}}$, the unit direction vector of the light source. A physical interpretation of this equation can be developed for the two-dimensional case when the light source and the viewing direction are in the same plane, as shown in Figure 1.

From Equation 1 it can be seen that to determine the velocity of the scattering object, the Doppler shift of the scattered laser light needs to be determined as well as having knowledge of the propagation direction of the light source and the direction from which the scattered signal is collected. While the light source and viewing direction can be determined through the experiment set-up, a method for determining the Doppler shift of the light frequency is needed. The PDV technique measures the shift in frequency directly using a frequency-to-intensity converter. Essentially, a filter that attenuates the scattered signal based on the frequency of that signal. For this filter to be efficient, it must have a cut-off that extends over the maximum expected range of velocities. For this a basic example of the expected performance of the filter can be developed. If a laser at a wavelength of $532 \mathrm{~nm}$ is used as the light source and the minimum to maximum expected velocity range is $+/-100 \mathrm{~m} / \mathrm{s}$ then the Doppler shift will be $+/-200 \mathrm{MHz}$ depending on $\hat{\mathbf{o}}$ and $\hat{\mathbf{i}}$. In this example the filter will then need to have a maximum cut-off of just over $400 \mathrm{MHz}$.

\footnotetext{
${ }^{1}$ r.p.tatam@cranfield.ac.uk; phone 441234754 630; fax 44 1234 752452; www.cranfield.ac.uk/sme/cpoe/cpoe.htm
} 


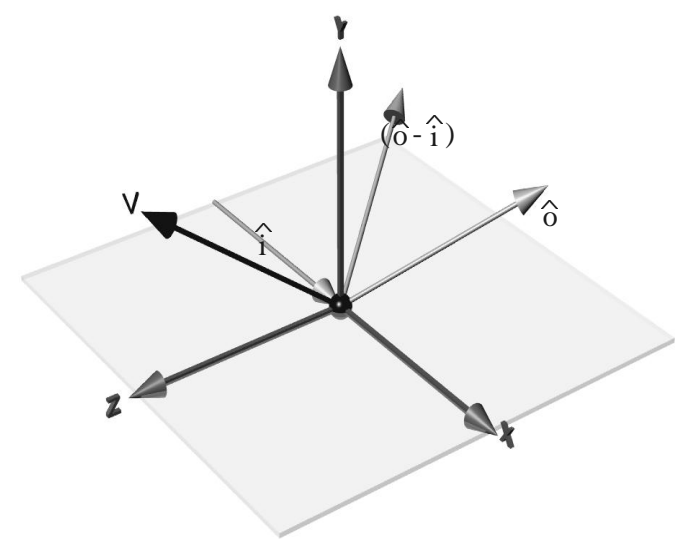

Figure 1. A schematic of the Doppler principle: V vector of the scattering particle, $\hat{\mathbf{i}}$ - light illumination vector, $\hat{\mathbf{o}}$ - observation vector, $(\hat{\mathbf{o}}-\hat{\mathbf{i}})$ sensitivity vector.

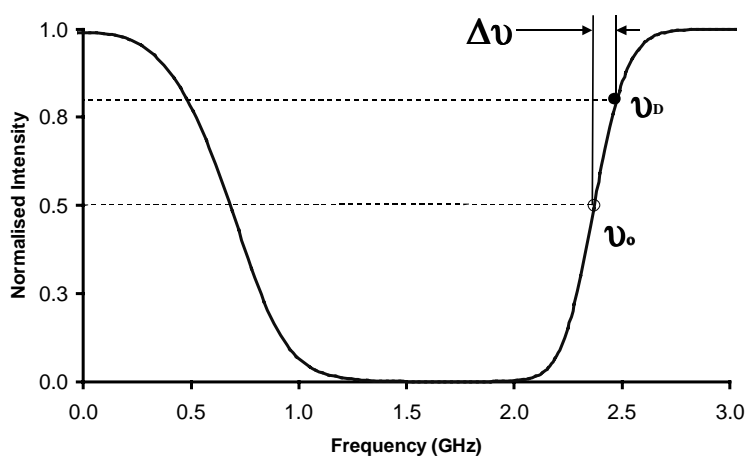

Figure 2. A frequency to intensity converter using an absorption band of iodine. The Doppler shift is measured as a change in intensity. $\Delta v=v_{\mathrm{D}}-v_{\mathrm{o}}$ is the difference between the Doppler shifted frequency $\left(\nu_{\mathrm{D}}\right)$ and the frequency of the light source $\left(v_{\mathrm{o}}\right)$

For such a narrow operation range Komine (1990) $)^{1}$ identified the use of the atomic and molecular absorption bands. These absorption bands are spectroscopic lines and are at specific frequencies that equate to the energy transfer levels of the substance used in the filter. Several substances have been identified that have suitable properties that could potentially be utilized ${ }^{2,3}$. Of these substances, iodine has an absorption band in the narrow tuning range of commercial Argon-ion laser and several absorption bands in the larger tuning range of Nd:YAG lasers ${ }^{2}$.

The philosophy of the PDV technique, showing in Figure 2 is to tune a narrow line-width laser light source to a point that is half way either up or down the side of an absorption band ${ }^{4}$. For no shift in the laser frequency, or zero velocity measured, the detector will see a signal of 50\% the value of full transmission. Any Doppler shift in the laser frequency will be measured as a change in the signal intensity level. The sign of the velocity direction of the measured component can also be determined from the direction of the shift to either lower or higher intensities.

Early in the development of PDV it was realized that to obtain usable data a reference signal was needed ${ }^{1}$. The reference signal split off before the filter is used to normalize the signal measured through the filter, correcting for variations in the scattered intensity. There are two sources that vary the intensity of the scattered signal. The power of the light source can vary in time as well as the scattering efficiency of the scattering medium. For measurement of flow velocities, a laser can be spread into a light sheet. The power distribution in this sheet can vary spatially across the sheet and in time. The flow is seeded with particles that are designed to follow the flow ${ }^{5}$. The amount of seeding and the size of the particles affect the scattering efficiency and hence the intensity of scattered signal. Hence, by normalizing the signal with a reference the effect of intensity variation due to laser power fluctuations and seeding density can be removed.

Other than the work of Roehle and coworkers ${ }^{6,7}$ all other systems reported in the literature follow the general scheme of a single laser source and multiple viewing directions. Initial work used CW lasers ${ }^{1,8}$. However, many research groups quickly moved to pulsed, frequency doubled $\mathrm{Nd}$ :YAG systems ${ }^{4,9}$ with the main aim of increasing the available laser power while at the same time making instantaneous velocity measurements possible. Meyers and Komine ${ }^{10}$ began the initial work in the area using a CW Argon-ion laser and their technique is perhaps the classic arrangement of PDV. Here, six cameras in three pairs of signal and reference cameras viewed a region of interest from three different directions, each pair utilizing its own iodine cell. Some of the reported difficulties with this system included the need to develop frame grabbers that could sample from two cameras at once (not commercially available at the time), integration of a network of computers to control the equipment, low signal to noise from the interlaced frame cameras used, differential sensitivity of the different iodine cells and the laser to vibration and temperature and the extensive development of software for control of the experiment as well as the processing of data. Other researchers have addressed some of these problems by changing the layout and philosophy of the equipment. To increase laser power 
and provide instantaneous measurements frequency doubled Nd:YAG lasers have been incorporated ${ }^{11}$. The introduction of digital technology has seen the use of CCD cameras and commercial frame grabbers that support multiple cameras. Cooled CCD cameras can also provide high signal to noise as well as higher data depths up to 16 $\mathrm{bit}^{12}$. These are expensive however, which has triggered the development of a number of novel arrangements to reduce the number of cameras used. One alternative has focussed on using a single camera to detect both the signal and reference images ${ }^{13}$. Viewing from three directions, this reduces the number of cameras from six to three at the expense of lower spatial resolution.

For any of the experimental arrangements, frequency drift of the laser is an important problem, especially for pulsed $\mathrm{Nd}$ :YAG systems. For these lasers an injection seeded laser is used to tune the host laser. The seed laser has a narrow line width $(\sim 5 \mathrm{kHz}) \mathrm{CW}$ laser at the fundamental frequency of the host laser $(1064 \mathrm{~nm})$. This seed laser, which is frequency tunable by changing the temperature of the cavity crystal, is introduced into the cavity of the host laser and because its power is orders of magnitude larger than the noise in the system, the host laser builds up preferentially around this frequency. To help lock the host laser to the seed laser the length of the cavity of the host laser is adjusted. This is done in a feedback manner with the position of one of the mirrors of the host being dithered. The resultant is an output that can be tuned over the gain curve of the host laser by tuning the seed laser and dithers about the frequency of the seed laser. The amount of dither is dependent on the setup of the whole laser system but can be of the order of $100 \mathrm{MHz}$. To counter this movement of the frequency of the host laser many research groups have employed a system to measure the frequency of the laser for the pulse that is used to take the measurement. A typical system is an arrangement of single point detectors and a separate iodine cell $^{4,14}$.

\section{THE CRANFIELD PDV SYSTEM}

The PDV system that has been developed at Cranfield University has been designed to address several of the problems and limitations of PDV systems that have been reported in the literature. Overall, the following criteria have focused the structure of the current PDV system. The PDV system will aim to;

- Measure the three components of velocity over a plane

- Be capable of both instantaneous and as time-average measurements

- Measure internal as well as external flows

- Minimize the number of cameras used

- Address problems of laser stability

- Address problems of stability of the iodine cell

To achieve these aims a system has been designed that uses the philosophy of one illumination direction and three viewing directions. A schematic of the experimental arrangement is shown in Figure 3. To achieve the aim of instantaneous measurement of velocity a injection seeded, frequency doubled, pulsed Nd:YAG laser is used (Spectra Physics GCR 190-30). This laser is capable of producing 300mJ @ 532nm in 10nsec pulses @ 30Hz, . A second CW laser (Lightwave Model 101) is coupled to and seeds the host laser at the host laser fundamental frequency of $1064 \mathrm{~nm}$. $\mathrm{I} / \mathrm{O}$ communication through the diagnostic port of the seed laser allows control of the seed laser frequency over a range of $20 \mathrm{GHz}$ as well as providing information on the seed laser temperature, the voltage being provided to the piezo-mirror and the build-up-time (BUT) error voltage. This information is used to assess the state and performance of the laser. The output from the host laser is frequency doubled to $532 \mathrm{~nm}$ using a second harmonic generator and this is used as the light source for the experiment. The laser pulse is spread into a light sheet using a combination of spherical and cylindrical lenses. For average velocity field measurements a tunable, single mode Argon ion laser can be used with the added benefit of a narrower laser line width.

A unique feature of this PDV system is that a single head is utilized consisting of two cooled CCD cameras (LaVision Imager3) and a single iodine cell. The cameras operate at $-15^{\circ} \mathrm{C}$ giving low noise performance. The arrays are 1280x1024 made up of square $6.7 \mu \mathrm{m}$ pixels and images collected are digitized to 12 bit. A single iodine cell is enclosed in a heated oven and iodine concentration in the cell is controlled by temperature control of a cold finger. Two PID controllers control the temperature of the oven and the cold finger to within $1{ }^{\circ} \mathrm{C}$. A schematic of the detector head is shown as a sub-figure in Figure 5. The imaging optics port an image into the head system that is first split into two paths with a 50/50 non-polarizing beam splitter. One path is directed to the reference camera via a turning mirror, this corrects the orientation of the image, while the second is directed through the iodine cell to the signal camera. 


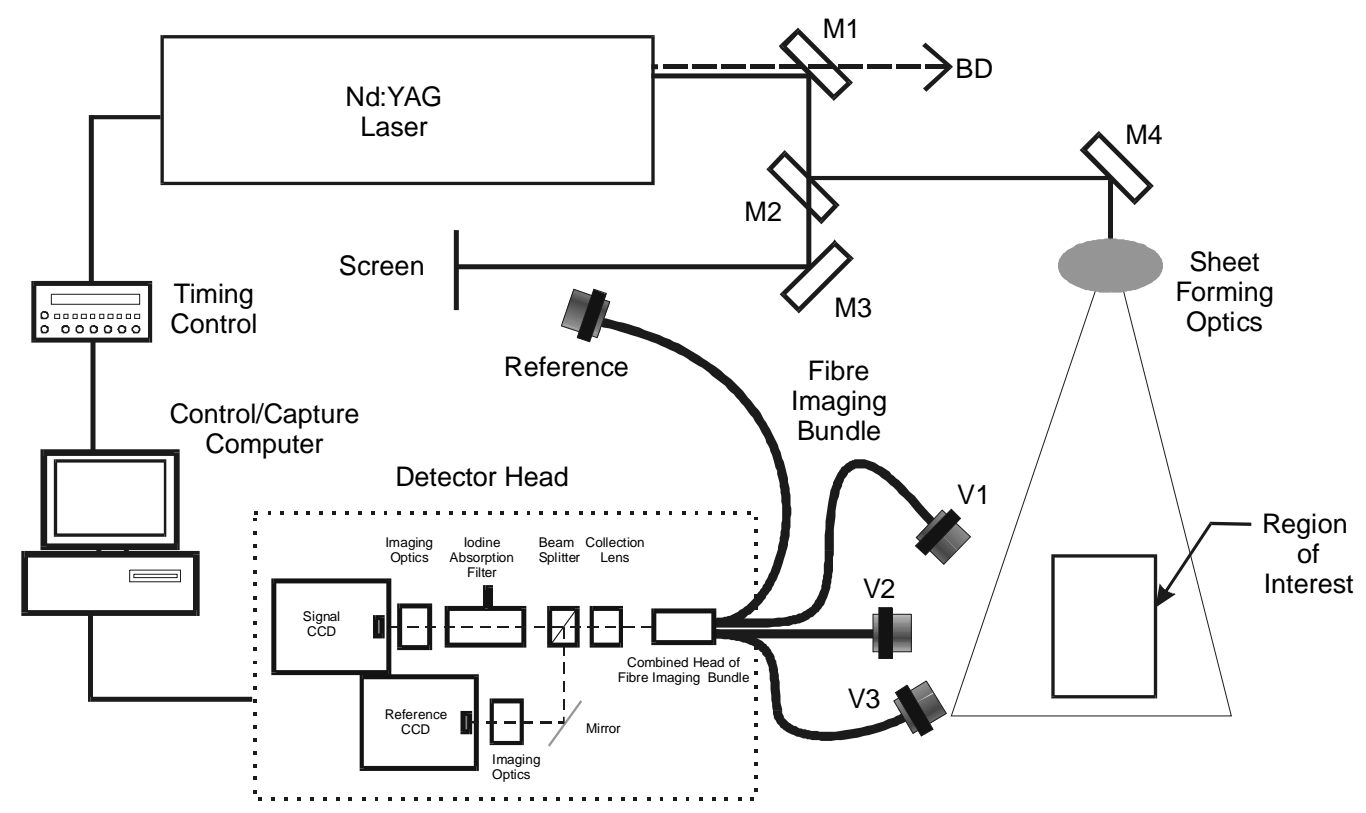

Figure 3. A schematic of the experimental arrangement for the PDV experiment. - 532nm beam; -- 1064nm beam; M1 and M2 - 532nm dicroic mirrors; M3 and M4 - turning mirrors, BD - 1064nm beam dump

The image coupled into the detector head is delivered to it by an imaging fiber bundle. This is a coherent array of fibers that is split into four channels. Three of these are used to view the same region of interest from three different directions. The fourth, as shown in Figure 3, images a small percentage of the laser pulse that has passed through a $532 \mathrm{~nm}$ dichroic mirror and scattered off a screen. This provides a means of monitoring the frequency of each laser pulse. To demonstrate the combining of channels an example image from the signal camera of the combined three views and the laser beam reference view is shown in Figure 4. The top left (View A) and bottom right (View B) and left (View C) are the three views of a reference target of crosses and views an area 200x $200 \mathrm{~mm}$. The perspective distortion of the target highlights the different directions of the three views. The top right corner of the image in Figure 4 is the fourth channel used to image the laser beam forming the laser frequency referencing system.

The use of the imaging fiber bundle reduces the total number of cameras used to two. The inclusion of the referencing channel allows the system to use only a single iodine cell. Imaging the pulse for frequency referencing also allows the distribution of laser power in the pulse to be recorded as well as the frequency distribution across the beam wave-front.

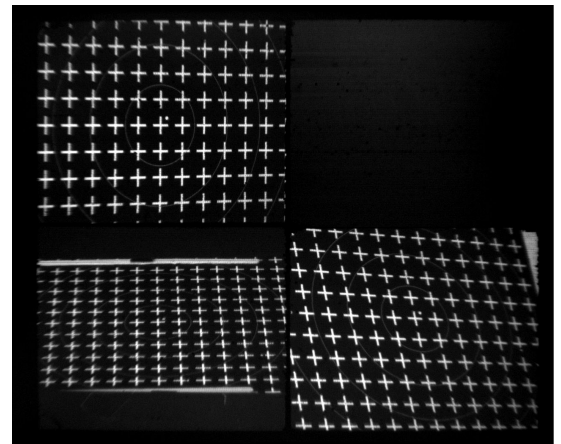

Figure 4. An example image of the view through the image bundle of a reference target captured with only one camera. The area viewed of the target is $200 \times 200 \mathrm{~mm}$. 


\section{THREE COMPONENT MEASUREMENT}

The majority of PDV systems reported in the literature have only been developed to the stage of measuring a single velocity component. It is surmised that a repetition of this single component system to measure the remaining two components will result in a full three-component PDV system. In the course of developing the full three-component Cranfield PDV system several problems in extrapolating a single component system have become evident. Of these two are discussed here. The first is the measurement of the view vector, $\hat{\mathbf{o}}$ for each pixel within the CCD and the second the effect of the transformation matrix on collected data used to map the results from the collected nonorthogonal coordinate system to an orthogonal coordinate system.

\subsection{Determination of the view angle $\hat{\mathbf{o}}$}

Measurement of $\hat{\mathbf{o}}$ can be determined by physically measuring the vector from the measurement volume to the detector and then normalising the vector. This is standard practice for most PDV systems reported in the literature ${ }^{10}$. However, this method has some limitations. The view vector $\hat{\mathbf{o}}$ as shown in Figure 1 is the bulk unit view vector for the system and this is only true for the central pixel of the two-dimensional array of a CCD camera. Each individual pixel (i,j) in the array views a different region of the plane under investigation and hence has a unique view vector, $\hat{\mathbf{o}}_{(\mathbf{i}, \mathbf{j})}$. This view vector is a function of the area viewed, the physical size of the array and the distance between the array and the viewed area. Accurate calculation of the view vector $\hat{\mathbf{o}}_{(\mathbf{i}, \mathbf{j})}$ for each individual pixel $(i, j)$, in the camera array is an important part of the full data processing procedure. Consider a configuration where a camera, $1 \mathrm{~m}$ from a plane is viewing a region of 100x100mm and is viewing perpendicularly. The variation in the view vector across the field is $\sim+/-6^{\circ}$. This would results in an error of $\sim 5 \%$ in the computed velocity if there is no correction for view angle.

Direct measurement of the bulk view vector is only suitable for situations where the distances involved are large, so that any measurement error does not introduce a significant error in the vector direction. After $\hat{\mathbf{o}}$ is determined, a correction to the view vector for each individual pixel can be achieved by assuming a simple pin-hole camera model ${ }^{15}$ of the view and correcting accordingly. Direct measurement of the bulk view vector is also difficult and potentially error prone in situations of restricted physical access or where the dimensions are small. Any error associated with the measurement of the bulk view vector will propagate through the calculation of all other view vectors. Use of the pinhole model of the view also limits the accuracy of the measurement of $\hat{\mathbf{o}}_{(\mathbf{i}, \mathbf{j})}$ by assuming that there are minimal optics used in the collection of the image and that the optical train does not significantly alter the view vector, through distortion of the image.

There is therefore a need for an algorithm that can determine $\hat{\mathbf{o}}_{(\mathbf{i}, \mathbf{j})}$ for each individual pixel that does not rely on physical measurement. There are several techniques presented in the literature that have been developed for the calibration of camera parameters. These camera calibration procedures determine the bulk camera intrinsic (i.e. focal length) and extrinsic (i.e. camera position) parameters and include methods that use a single view of a target from a single camera ${ }^{16}$, multiple views of a target from a single camera ${ }^{16,17}$ and multiple views from a number of cameras ${ }^{16}$. The procedures process images of test targets or images that have know points located within the field of view. A limitation of these techniques is that only bulk parameters are calculated, hence only the bulk view vector, $\hat{\mathbf{o}}$ is found. Individual correction of each pixel for view angle is again needed after the bulk parameters are derived.

Techniques like PDV are unique in that they measure over a plane defined by the propagation of the expanded laser beam into a sheet. For multi-component measurement, multiple views of the region-of-interest (ROI) are collected and mapped onto a single co-ordinate system oriented with the experiment ${ }^{9,10}$. This requires the de-warping of individual views onto the pre-defined plane and allows the interpolation of different measured components onto the same spatial grid. A calibration procedure used to determine the de-warping mapping functions using a test target of co-planar location markers that are at a known spacing is often employed ${ }^{9,10}$. The de-warping procedure corrects the view for image perspective distortion as well as radial distortion and aberrations introduced by optical components within the imaging system. The mapping function therefore carries information about the bulk view vector to the target as well as for each pixel in the array and information about any local change in the view vector introduced by local distortion of 
the know target image. A procedure ${ }^{18}$ has been developed to determine $\hat{\mathbf{o}}_{(\mathbf{i}, \mathbf{j})}$ using the information of the view distortion determined in the de-warping procedure.

To test the procedure an image of a test target consisting of a regular array of co-planar crosses was generated using a $3 \mathrm{D}$ drawing package ${ }^{19}$. This allowed manipulation of the view of the target to a know angle. Camera parameters, including the position of the camera and camera view orientation can be explicitly included into the target model. The package also allowed lens modelling of the view to generate perspective distortion using a pin-hole camera model. An image of a suitable target with crosses spaced at a known regular interval was generated with a known view direction. For this image the target has been rotated about the $\mathrm{X}$-axis by $30^{\circ}$ with the top of the target tilted towards the camera. To generate the target, dimensionless length units in a ratio that might be typical of an experiment where used. Arbitrary units are used as length scales. The camera is located at $\mathrm{P}_{\text {cam }}=(0,0,1000)$ in the reference frame of the experiment and views a region 100x100 units. The view is generated by modelling a lens with a focal length of 250 units. The crosses are at a regular spacing in the $\mathrm{X}$ and $\mathrm{Y}$ directions of 10 units and have a line aspect ratio of 6:1. A significant advantage of computer generating the image is that there is no noise in the image and the image is in perfect focus.

Using the view angle determination technique ${ }^{18}$ the spatial variation in the mapping function has been used to determine the direction cosines of the vector $\hat{\mathbf{o}}_{(\mathbf{i}, \mathbf{j})}$ at each pixel location, (i,j) in the image. Maps, the same size (number of pixels) as the de-warped image, of the magnitude of the components of the direction cosines are shown in Figure 5 . The derivation of the direction cosines based on the spatial gradient of the mapping function does not determine the sign of the individual components. Using the derived rules about the view ${ }^{18}$ the sign of the components can be determined. It can be seen in Figure 5 that for the case of a pure rotation about the X-axis only the sign direction of the $\hat{\mathbf{o}}_{\mathbf{x}(\mathbf{i}, \mathbf{j})}$ component is altered.

A simple pin-hole camera model ${ }^{17}$ of the view of the target can be used to investigate the accuracy of the determined view angle along the major axes of the image. Angles can be calculated along the major axis using the following:

$$
\begin{gathered}
\alpha_{x(i, 0)}=\tan ^{-1}\left(\frac{\boldsymbol{f}}{\mathrm{i}}\right) \\
\alpha_{y(0, j)}=\tan ^{-1}\left(\frac{\boldsymbol{f} \cdot \cos (\theta)}{\boldsymbol{f} \cdot \sin (\theta)-\mathrm{j}}\right) \\
\alpha_{\mathrm{z}(0, \mathrm{j})}=\tan ^{-1}\left(\frac{\boldsymbol{f} \cdot \sin (\theta)-\mathrm{j}}{\boldsymbol{f} \cdot \cos (\theta)}\right)
\end{gathered}
$$

Here, the bulk view angle, $\theta$ is the angle of rotation about the $\mathrm{X}$-axis and $f$ is the focal length from the origin of the plane to the camera. Several values of $\theta$ in steps of $10^{\circ}$ have been use in this test from $\theta=0^{\circ}$, which is a perpendicular view of the target to $\theta=60^{\circ}$ which is near the practical extent of what would be used in an experiment. The variation of $\alpha_{x}, \alpha_{y}$ and $\alpha_{z}$ in degrees along the major axes is shown in Figure 6 for these test cases. The procedure to determine the view shows excellent agreement with the simple pin-hole camera model of view angle components for a few bulk angles in the range $10^{\circ}-60^{\circ}$. There is some variation in the gradient seen in the plot, however the error in the difference between the two results is less than $0.75 \%$. The technique breaks down for bulk view angles that are below $10^{\circ}$, which are views that are close to a perpendicular view. In experiment, the views used in PDV are often away from the perpendicular in the range $20^{\circ}-50^{\circ}$. Hence in practical use, the technique will resolve the view angle for each individual pixel in the view. 


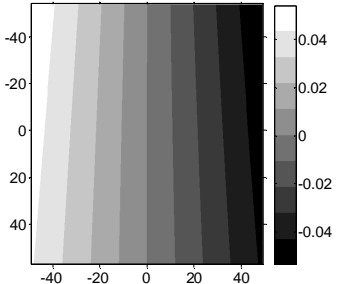

(a)

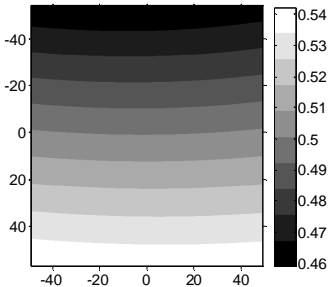

(b)

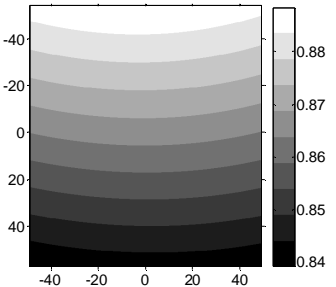

(c)

Figure 5. The distribution of the magnitude (grey-scale) of the signed direction cosines; (a) $\hat{\boldsymbol{o}}_{(i, j) \boldsymbol{x}}$ (b) $\hat{\boldsymbol{o}}_{(\boldsymbol{i}, \boldsymbol{j}) \boldsymbol{y}}$

$\hat{\boldsymbol{o}}_{(i, j) z} \cdot$ (Axes in arbitrary length units)

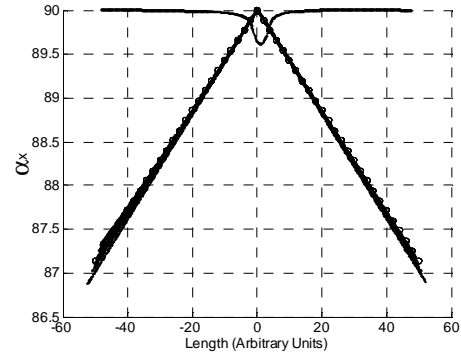

(a)

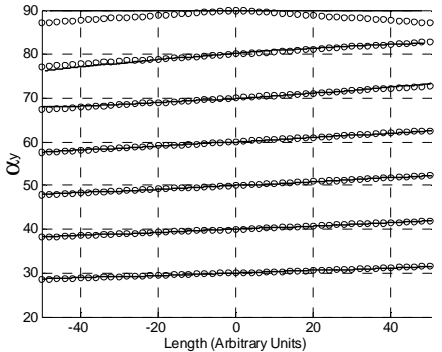

(b)

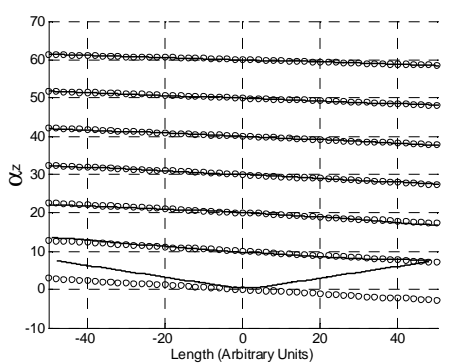

(c)

Figure 6. The distribution of the calculated view angle $(\mathrm{O})$ compared to the angle determined using a pin-hole camera model (-): (a) angle $\alpha_{x}$ along the $\mathrm{X}$ axis (b) angle $\alpha_{\mathrm{y}}$ along the $\mathrm{Y}$ axis and (c) angle $\alpha_{\mathrm{z}}$ along the $\mathrm{Z}$ axis. (Abscissa in arbitrary length units)

\subsection{Mapping of results}

There have been reported in the literature several single component configurations of PDV that could be expanded to full three component configurations. The majority have been of the type where a region of interest is illuminated by a single laser sheet and viewed from up to three directions. One research group ${ }^{7}$ has used the complementary configuration where a single view is used to interrogate the region of interest that has been illuminated consecutively from three different directions. An analysis of typical configurations that could be expected in an experiment and some that have been reported has been carried out. The focus of the analysis is of different configurations of laser sheet(s) and view(s) described in Table 1 which each have different sensitivity vectors.

How well the measured non-orthogonal components in the direction of the sensitivity vectors can be mapped to the orthogonal Cartesian co-ordinate system can be described by the value of the conditioning number of the transformation matrix. The conditioning number $\kappa(\mathbf{A})$, of a matrix can be used as a measure of the sensitivity of the solution of the system of linear equations defined by the matrix to errors in the data. The condition number is defined as ${ }^{20}$,

$$
\kappa(\mathbf{A})=\|\mathbf{A}\| \mathbf{A}^{-1} \|
$$

Here, $\mathbf{A}$, is the transformation matrix and $\|\mathbf{A}\|$ is the norm of the matrix and can be defined in several different forms. In this analysis the norm is based on the largest column sum in $\mathbf{A}$.

The computed conditioning number for each of the configurations is given in Table 1. The ideal case, Configuration F, of the three sensitivity vectors being aligned with the axis of the Cartesian co-ordinate system gives a minimum value for $\kappa(\mathbf{A})$ for the different definitions. The closer the value of $\kappa(\mathbf{A})$ to this ideal case the better conditioned the matrix is for transforming measured results from one co-ordinate system to another. 
Table 1. A comparison of different configurations and resultant sensitivity vectors of the computed matrix conditioning number for different definitions.

\begin{tabular}{|c|c|c|c|c|}
\hline \multirow[b]{2}{*}{ Description } & \multicolumn{3}{|c|}{ Sensitivity Matrix } & \multirow[b]{2}{*}{$\kappa$} \\
\hline & $\left(\hat{\mathbf{o}}_{1}-\hat{\mathbf{i}}\right.$ & $\left(\hat{\mathbf{o}}_{2}-\hat{\mathbf{i}}\right)$ & $\left(\hat{\mathbf{o}}_{3}-\hat{\mathbf{i}}\right)$ & \\
\hline \multirow{3}{*}{$\begin{array}{l}\text { Configuration A: three views of a single laser light sheet from the } \\
\text { positive } \mathrm{Z} \text { side. Each view is a function of only two of the } \\
\text { Cartesian co-ordinate variables. }\end{array}$} & -0.707 & 0.500 & 0.500 & \multirow{3}{*}{14.750} \\
\hline & -0.707 & -0.500 & 0.500 & \\
\hline & -0.924 & 0.000 & 0.383 & \\
\hline \multirow{3}{*}{$\begin{array}{l}\text { Configuration B: Similar to A, however the aim of the set-up is to } \\
\text { maximize the angle between views while not introducing a large } \\
\text { perspective distortion to the view of the region of interest. }\end{array}$} & -0.500 & 0.707 & 0.500 & \multirow{3}{*}{7.110} \\
\hline & -0.500 & -0.707 & 0.500 & \\
\hline & -0.924 & 0.000 & 0.383 & \\
\hline \multirow{3}{*}{$\begin{array}{l}\text { Configuration C: Follows the set-up of Roehle } \text { etal }^{7} \text {; a single view } \\
\text { and three laser sheets in the same plane from orthogonal } \\
\text { directions. }\end{array}$} & -0.707 & 0.000 & 0.707 & \multirow{3}{*}{4.500} \\
\hline & 0.707 & 0.000 & 0.707 & \\
\hline & 0.000 & 0.707 & 0.707 & \\
\hline \multirow{3}{*}{$\begin{array}{l}\text { Configuration D: Similar to Configuration B except that one view } \\
\text { has been rotated by } 180^{\circ} \text { about the Y-axis. }\end{array}$} & -0.500 & 0.707 & 0.500 & \multirow{3}{*}{3.284} \\
\hline & -0.500 & -0.707 & 0.500 & \\
\hline & -0.383 & 0.000 & -0.924 & \\
\hline \multirow{3}{*}{$\begin{array}{l}\text { Configuration E: The reported sensitivity vectors of Reinath }{ }^{11} \text {. } \\
\text { This is similar to Configuration D. }\end{array}$} & -0.460 & 0.628 & 0.628 & \multirow{3}{*}{3.876} \\
\hline & -0.460 & -0.628 & 0.628 & \\
\hline & -0.383 & 0.000 & -0.924 & \\
\hline \multirow{3}{*}{$\begin{array}{l}\text { Configuration } F \text { : The ideal case of all three sensitivity vectors } \\
\text { being aligned with the orthogonal Cartesian co-ordinate system. }\end{array}$} & 1.000 & 0.000 & 0.000 & \multirow{3}{*}{1.000} \\
\hline & 0.000 & 1.000 & 0.000 & \\
\hline & 0.000 & 0.000 & 1.000 & \\
\hline
\end{tabular}

Low values of $\kappa(\mathbf{A})$ are achieved for configurations where the sensitivity vectors are well spread. This is evident for configurations where one of the sensitivity vectors is on the opposite sides to the light sheet (Configurations D \& E) compared to the having all three sensitivity vectors on the same side (Configurations A, B \& C).

There is a small change in view direction vectors in Configuration A to Configuration B. This however leads to a halving of $\kappa(\mathbf{A})$. A further $50 \%$ reduction can be achieved on the value of Configuration B by moving one of the view directions to the other side of the light-sheet as in Configuration D.

Other definitions of $\kappa(\mathbf{A})$, where the norm of the matrix is based on some other definition show a similar trend in the magnitude of $\kappa(\mathbf{A})$ for the same configuration. Low values of $\kappa(\mathbf{A})$ are obtained for conditions where the sensitivity vectors are well spread or are close to orthogonal and are close to the directions of the Cartesian co-ordinate system of the experiment. High values of $\kappa(\mathbf{A})$ are determined where the sensitivity vectors have similar direction. For PDV, the separation of sensitivity vectors $(\hat{\mathbf{o}}-\hat{\mathbf{i}})$ can be close even though the separation of the view vectors $(\hat{\mathbf{o}})$ are well spread as seen in Configurations A \& B.

To investigate the effect of the transformation matrix on error propagation an ideal velocity field has been calculated from which three non-orthogonal components have been calculated. The velocity field is originated with respect to an orthogonal co-ordinate system and has unit component velocity field in the direction of each of the axes such that $(\mathbf{U}, \mathbf{V}, \mathbf{W})=[1,1,1]$. While the resultant vector is not a unit vector, this field does aid in the assessment of individual components.

The three computed components of the velocity field for each of the three sensitivity vectors have been calculated using the Doppler formula for the configurations listed above. In each case the region of interest defined by the laser sheet(s) has been oriented in the X-Y plane for this investigation. The direction of the laser sheets are parallel to either the $\mathrm{X}$ or $\mathrm{Y}$ axis and the rays within the sheet are parallel (not diverging as would typically be found in an experiment). The view direction, $\hat{\mathbf{o}}_{(\mathbf{i}, \mathbf{j})}$ varies over the two-dimensional region of interest due to perspective viewing. As part of the calculation of the non-orthogonal components a pin-hole camera model of the view has been used to calculated the different views due to perspective. 
To facilitate the modelling, some arbitrary values have been used and these have been chosen to be similar to those found in a typical experiment. A view of $200 \times 200 \mathrm{~mm}$ has been used and each view camera position is $1.5 \mathrm{~m}$ from the region of interest. In the Doppler calculation to determine the Doppler shift $(\Delta v)$ the laser wavelength is assumed to be $532 \mathrm{~nm}$.

Mapping of data, calculated as described above, from a non-orthogonal co-ordinate system to the orthogonal coordinate system aligned with the experiment for pristine data results in the recovery of the original velocity field used in the calculation. The only noise in the system is due to round-off errors in the calculations. To simulate a situation typically found in experimental data, a known amount of noise is added to the orthogonal components before the nonorthogonal components are computed. For each condition, random noise up to $10 \%$ of the maximum value has been added. For this example the noise has also been computed to have a Gaussian distribution. The distribution of the added noise before the transformation can be seen in Figure $7(\mathrm{a})$ and has a range of $+/-0.05 \mathrm{~m} / \mathrm{s}$. The profile of the added noise is plotted in each of the probability distribution function (PDF) plots shown in Figure 7. The PDF's are made up of the local determined velocity minus the known velocity at that point. Plotted with the added noise is the $\mathrm{PDF}$ of the noise after the transformation from the non-orthogonal to orthogonal co-ordinate system.

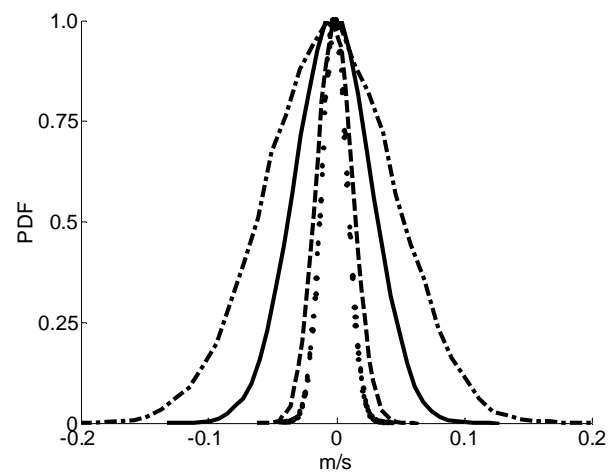

(a) Configuration $\mathrm{A}$

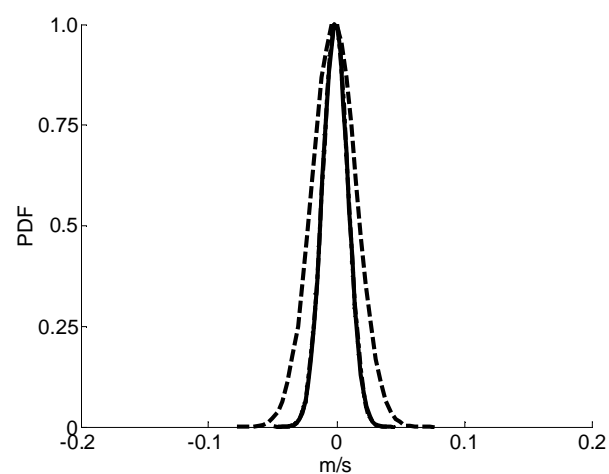

(c) Configuration $\mathrm{C}$

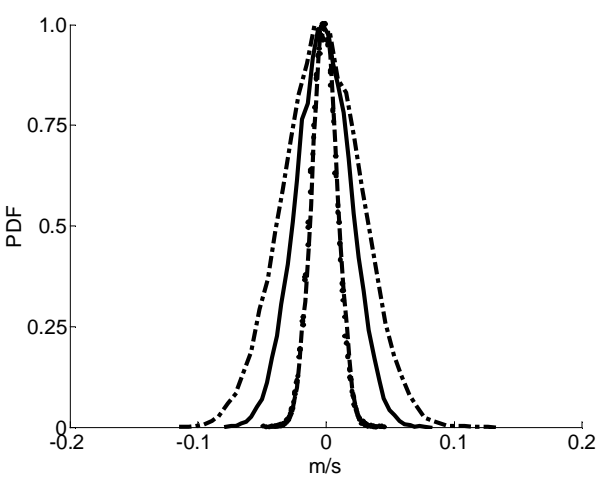

(b) Configuration B

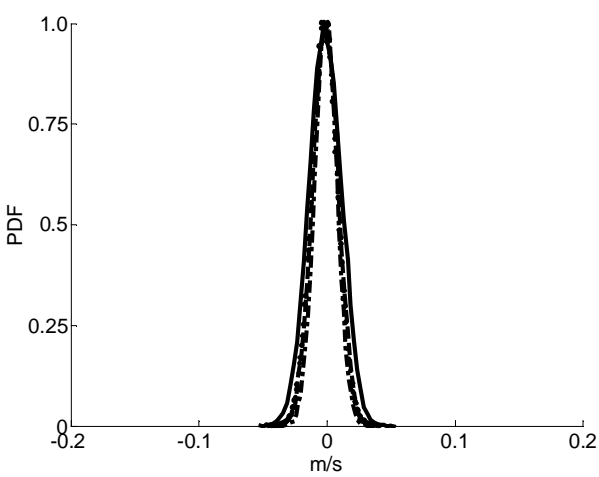

(d) Configuration D

Figure 7. The effect of the transformation matrix on introduced errors.

....... Added noise; __ U component;-- - V component; - - - . W component

Figure 7(a) shows the effect of a transformation matrix with a high value of $\kappa(\mathbf{A})$ on the added noise for Configuration A where all three sensitivity vectors are close together. The noise in each of the components is significantly amplified with a factor of 4 for the $\mathbf{W}$ component. There is a noticeable reduction in the amplification of the noise for Configuration $\mathrm{B}$ where there is a slight spreading of the sensitivity vectors compared to Configuration A. The result of 
using a single view and three laser sheets, Configuration $C$, is a low value of $\kappa(\mathbf{A})$ and only amplification of noise for one component. Only one of the views is sensitive to this component, $\mathbf{V}$, while the other components have two or more views contributing to the $3-\mathrm{D}$ vector. Imaging both sides of a single laser sheet as in configuration $\mathrm{D}$ results in a well condition matrix and only small amplification of the noise present in the original data set.

The results of this simulation indicate that even if there is only a small amount of noise in the collected component, there can be significant amplification of that noise if the sensitivity components are close together. The condition number of the transformation matrix can be used as an indication as to what extent any noise will be amplified.

\section{EXAMPLES OF VELOCITY MEASUREMENTS}

In the development stage of the PDV system a rotating disc has been used to provide a moving field of a well-defined velocity. The data presented here are the processed results collected from a rotating disc of 200mm diameter that has been coated with flat white paint with the aim to give near uniform reflection. The disc is illuminated by the laser at an angle that is near perpendicular to the rotational axis. The three views are arranged so that all three are sensitive to the rotating field and each view is captured using a $35 \mathrm{~mm}$ SLR lens. As the wheel is not transparent, all three views are on one side of the laser sheet and are arranged according to Configuration B.

The computed velocity components from each of the three views are shown in Figure 8. Scaling factors determined in the de-warping procedure are used to scale the data's physical dimensions within the image. The banded grey scale colour map shows the magnitude of the instantaneous velocity component of the wheel measured by each view. The regular change in the banded grey scale colour map indicates the expected regular change in velocity component of the disc. From zero velocity at the center of the disc to a maximum at the extent of the disc and at each side the velocity is in opposite directions.

These three components of the disc's velocity have been measured in a non-orthogonal reference frame. The data can be transformed to an orthogonal coordinate system that is aligned with the experiment using an appropriate transformation matrix. This $3 \times 3$ matrix is unique for each point in the measurement plane ${ }^{9}$. In Figure 9 the mapped data is displayed as a vector map showing the in-plane velocity component at every $8^{\text {th }}$ point in the field. The pattern of the vectors in the map show the center of the rotating disc, the radial increase in velocity magnitude and the direction of rotation of the disc. All of these are as expected of this velocity field. The results show the ability of this PDV system to detect velocities in the $+/-30 \mathrm{~m} / \mathrm{s}$ range, resolving well the magnitude and direction of velocity.

In Figure 10 the PDV system has been applied to the measurement the velocity field of a jet exiting a round nozzle. This result is an average velocity field of the flow and has been captured using an Argon ion laser. This jet has a smooth contraction nozzle inlet, $20 \mathrm{~mm}$ in diameter and can be expected to generate a 'top-hat' velocity profile at the nozzles exit. The jet flow and surrounds where seeded with $0.6 \mu \mathrm{m}$ particles and the seeded flow was evacuated from the area using an extraction hood. This was located 75 jet diameters above the nozzle exit.

Plotted in Figure 10 are velocity vectors of the first four diameters of the jet showing the presence of the jet's potential core. (Radially every $2^{\text {nd }}$ vector is plotted and axially every $10^{\text {th }}$ vector is plotted) With increasing axial distance, there is evidence that the jet's shear layer is eroding the potential core. However the potential core is still evident at the extent of the plot where on the jet centerline vector magnitudes are similar to those at the nozzle exit.

Less stringent requirements on the performance of the imaging optics in imaging the seeded particles will allow the PDV technique to be used in previously restricted situations. For example, exchange of the image capture optics similar to the borescope will allow the application of this PDV technique to investigate internal flows, for example turbomachinary, where viewing access is restricted even though the collected image has significant perspective distortion. 


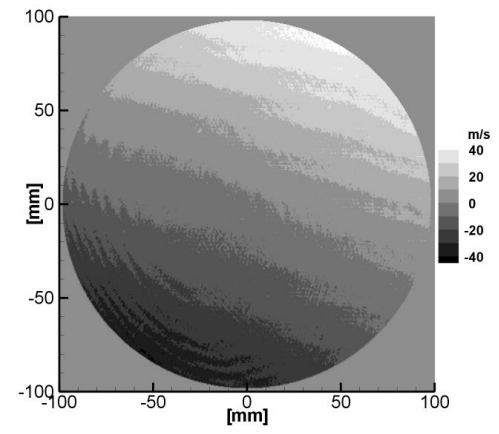

View A

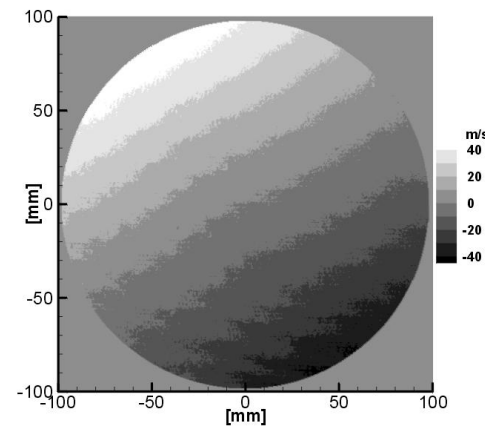

View B

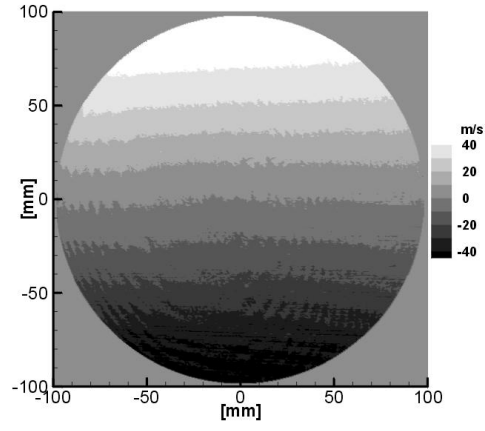

View C

Figure 8. The computed three non-orthogonal components measured by the three different views.

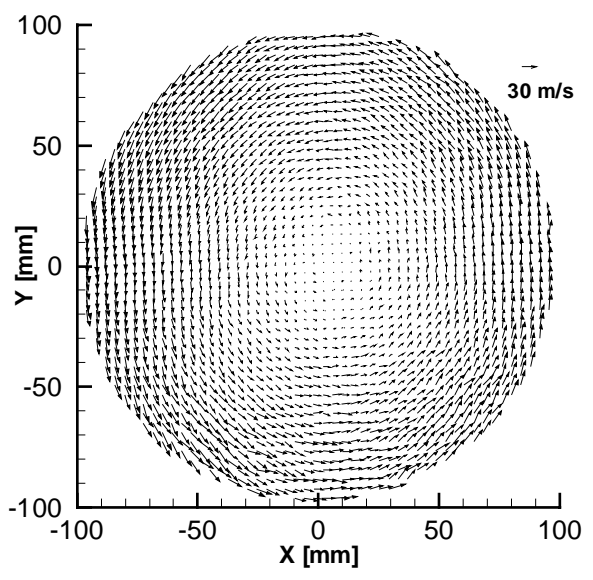

Figure 9. A vector map of the velocity field of the rotating disk

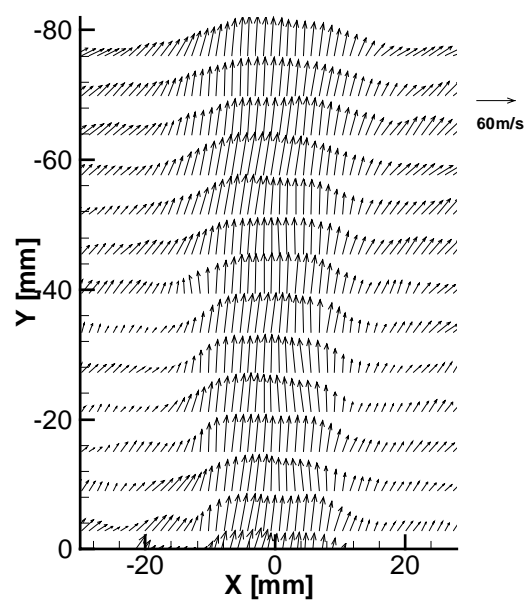

Figure 10. The velocity field of a jet exiting from a round smooth contraction nozzle.

\section{CONCLUSIONS}

A three component PDV system based on imaging a light sheet from three directions using imaging fibre bundles has been described. The system is capable of measuring three instantaneous components of velocity over a planar region. In developing the full three-component capability of the system, two major difficulties are apparent and these are described. The determination if each individual view vector from the region of interest to the camera is required for the Doppler calculation. A technique is demonstrated that does not require the physical measurement of the vector but uses information determined in the de-warping of the view to calculate each individual view vector. For three component PDV, all data is captured some non-orthogonal measurement system. It is highlighted that if the views are too close together any small error in the captured data can be amplified when transformed from the non-orthogonal to an orthogonal co-ordinate system. The condition number of the matrix can be used to assess the influence of the transformation matrix on amplification of errors. If the condition number is small, there will be minimal amplification of errors. The system is demonstrated by measuring the velocity field of a rotating wheel and of a round jet. 
1. Komine,H. US Pat No. 4,919,536, 1990.

2. Miles, R.B., Yalin, A.P., Tang, Z., Zaidi, S.H., and Forkey, J.N. "Flow field imaging through sharp-edged atomic and molecular 'notch' filters", Meas. Sci. Technol. Vol 12. pp. 442-451, 2001.

3. Chan,V.S.S., Heyes, A.L., Robinson, D.I. and Turner, J.T. "Iodine absorption filters for Doppler global velocimetry", Meas. Sci. Technol. Vol 6, pp 784-794, 1995.

4. Elliot, G.S. and Beutner, T.J. "Molecular filter based planar Doppler velocimetry", Prog in Aero. Sci. Vol 35 pp 799-845, 1999.

5. Meyers, J.F., "Generation of Particles and Seeding," von Karman Institute Lecture Series 1991-08, 1991.

6. Roehle, I. "Three-dimensional Doppler global velocimetry in the flow of a fuel spray nozzle and in the wake region of a car", Flow Meas. Instrum., Vol.7, No3/4 pp 287-294, 1996.

7. Roehle, I., Schodle, R., Voigt, P. and Willert, C. "Recent developments and applications of quantitative laser light sheet measuring techniques in turbomachinery components", Meas. Sci. Technol. Vol.11 pp 1023-1035, 2000.

8. Ford, H.D. and Tatam, R.P. "Development of extended field Doppler velocimetry for turbomachinary applications", Optics and Lasers in Engineering, Vol 27 pp 675-696, 1997.

9. Nobes, D.S., Ford, H.D. and Tatam, R.P. "Instantaneous, three-component planar Doppler velocimetry using imaging fibre bundles" Expt. In Fluids, 2003.

10. Meyers J.F., and Komine H. "Doppler global velocimetry: a new way to look at velocity", Laser Anemometry, 1, 289-296, 1991.

11. Reinath, M.S., "Doppler Global Velocimeter Development for the Large Wind Tunnels at Ames Research Center", NASA TM-112210, 1997.

12. Meyers, J.F., Fleming, G.A., Althoff Gorton, S. and Berry, J.D., "Instantaneous Doppler Global Velocimetry Measurements of a Rotor Wake: Lessons Learned", 9th International Symposium on Applications of Laser Techniques to Fluid Mechanics, Lisbon, Portugal, July 13-16, 1998.

13. Smith, M., Northam, G., and Drummond, P., "Application of Absorption Filter Planar Velocimetry to Sonic and Supersonic Jets," AIAA Journal, Vol. 34, No. 3, pp. 434 - 441, 1996.

14. Mosedale, A.D., Elliott, G.S., Carter, C.D. and Beutner, T.J. "Planar Doppler velocimetry in a large-scale facility", AIAAJ, Vol 38, No6 pp 1010-1024, 2000.

15. A. Gruen and T.S. Huang (Eds) "Calibration and Orientation of Cameras in Computer Vision", Springer-Verlag Press 2001.

16. R.Y. Tsai, "A versatile camera calibration technique for high-accuracy 3D machine vision metrology using off-theshelf TV cameras and lenses", IEEE J Robotics and Automation, RA-3(4), 323-344, 1987.

17. Z. Zhang, "A flexible new technique for camera calibration" IEEE Trans. Pattern Analysis and Machine Intelligence, 22(11), $1330-1334,2000$.

18. Nobes, D.S., Wieneke, B. and Tatam, R.P. "Determination of view vectors from image warping mapping functions", Optical Engineering. In Press, 2003.

19. Rhinoceros "NURBS modelling for windows" http://www.rhino3d.com/

20. Kreysig, E. Advanced Engineering Mathematics. $7^{\text {th }}$ ed. Wiley, New York, 1993. 


\section{Cranfield University}

CERES https://dspace.lib.cranfield.ac.uk

School of Aerospace, Transport and Manufacturing (SATM)

Staff publications (SATM)

2003-08-03

Planar Doppler velocimetry measurements of flows using imaging fibre bundles

Nobes, David S.

SPIE - International Society for Optics and Photonics

David S. Nobes, Helen D. Ford and Ralph P. Tatam

http://dx.doi.org/10.1117/12.505842

Downloaded from Cranfield Library Services E-Repository 